\title{
FPRI mediates the tumorigenicity of human cervical cancer cells
}

This article was published in the following Dove Press journal: Cancer Management and Research

\section{Guangming Cao Zhenyu Zhang}

Department of Obstetrics and Gynecology, Beijing Chao-Yang Hospital, Capital Medical University, Beijing, China
Correspondence: Zhenyu Zhang Department of Obstetrics and Gynecology, Beijing Chaoyang Hospital, Affiliated to Capital Medical University, 8 Workers' Stadium South Road, Chaoyang, Beijing 100020, China Tel/fax +86 $8523 \quad 1507$ Email zhenyuzhang2018@163.com
Purpose: The present study aimed to investigate the role of FPR1 and the downstream effectors such as NF- $\kappa B$ and IL-6/8 in the development of cervical cancer.

Patients and methods: FPR1 protein expression was detected via immunohistochemical staining in tissue microarrays containing cervical cancer tissues from 185 patients. Following FPR1 silencing in SiHa cells using lentiviral siRNA delivery, biological characteristics and tumor formation were evaluated in vitro and in vivo, respectively. Phosphorylated NF- $\kappa B$ levels were detected by Western blotting, while IL-6 and IL- 8 secretion were detected by ELISA in both FPR1 knockdown and control SiHa cells. Human umbilical vein endothelial cell tube formation assays were performed to evaluate the angiogenesis-promoting ability of IL-6 and IL-8 secretion in FPR1 knockdown and control SiHa cells. Neovascularization, proliferation and apoptosis markers were detected by immunohistochemical staining to analyze the tumorigenic role of FPR1.

Results: Immunohistochemistry of cervical cancer tissues from 185 patients revealed high FPR1 expression levels in patients with advanced-stage disease and/or poor prognosis. Compared with control cells, cervical cancer cells in which FPR1 was silenced exhibited inhibition of cell invasion, migration and proliferation and higher levels of apoptosis. NF- $\kappa \mathrm{B}$ was inhibited in FPR1 knockdown in SiHa cells. IL-6/8 upregulation by FPR1 activation stimulated angiogenesis. FPR1 deficiency inhibited the tumorigenicity of cervical cancer cells in nude mice. FPR1, IL-6, IL-8, CD31 and Ki67 levels were all reduced, whereas cleaved caspase- 3 was upregulated, in the FPR1 knockdown group compared with the levels in the control group.

Conclusion: High FPR1 expression was associated with advanced stage and poor prognosis in cervical cancer patients. FPR1 activation induced NF- $\kappa$ B nuclear translocation to promote cervical cancer development through the upregulation of IL-6 and IL-8 expression. Inhibiting FPR1 activity may thus have potential therapeutic value in cervical cancer patients.

Keywords: cervical cancer, FPR1, IL-6, IL-8

\section{Introduction}

Cervical cancer is the second most common malignant tumor of the female reproductive system. ${ }^{1}$ Despite the widespread use of the Thinprep Cytology Test and HPV screening, as well as the development of treatments, cervical cancer remains one of the most common causes of cancer-related death in developing countries. ${ }^{2}$ Although early cervical cancer can be cured and has a good prognosis through surgery and/or radiation with or without chemotherapy, effective treatments for advanced, recurrent or persistent cervical cancer are lacking. ${ }^{3}$ In addition, complications and severe side 
effects are not uncommon following surgery, chemotherapy and radiotherapy, which are the main treatments currently. Therefore, it is vital to find novel therapeutic targets for cervical cancer. ${ }^{4}$

FPR1 is a G-protein-coupled 7 transmembrane cell surface receptor (GPCR) involved in inflammation, wound healing and antimicrobial host defense. ${ }^{5,6}$ Accumulating evidence demonstrates that FPR1 contributes to tumor progression by mediating tumor cell chemotaxis and proliferation as well as by promoting inflammation. ${ }^{5,7-9}$ FPR1 activation has been shown to induce signaling pathways related to chronic inflammation, such as ERK, MAPK, NF- $\mathrm{BB}$ and STAT3, in malignant glioma cells, colon cancer and hepatocellular carcinoma cells. ${ }^{10-14}$ However, the role of FPR1 in tumorigenesis remains poorly understood, especially in cervical cancer. In the present study, immunohistochemistry (IHC) was performed on cervical cancer tissue microarrays (TMAs) to detect FPR1 expression levels. High FPR1 expression levels were detected in cervical cancer patients with advanced-stage disease and/or poor prognosis. These results suggested that FPR1 may participate in the development of cervical cancer by regulating inflammation.

Chronic inflammation is involved in the development of a wide variety of tumors. ${ }^{15,16}$ In cervical cancer patients, IL-6 and IL-8 concentrations in cervicovaginal washings are significantly higher than those in cervical intraepithelial neoplasia patients and controls. ${ }^{17-19}$ In most patients, local IL-6 and IL-8 levels were also higher in serum. ${ }^{18}$ Multiple cervical cancer cell lines have been shown to synthesize and secrete IL-6/8 in vitro. ${ }^{20,21}$ IL- 6 and IL- 8 are multifunctional cytokines that regulate inflammation and cancer development. ${ }^{20,22-24}$ Increasing evidence demonstrates that IL-6/8 plays a vital role in the pathogenesis of cervical cancer.

The present study aimed to investigate whether FPR1 activation can promote IL- 6 and IL- 8 secretion via NF- $\kappa B$ signaling during cervical cancer development.

\section{Patients and methods}

\section{Cell culture}

Cervical cancer cell lines (SiHa and HeLa cells) and human umbilical vein endothelial cells (HUVECs) were obtained from the Medical Research Center of Beijing Chaoyang Hospital. SiHa and HeLa cells were cultured in RPMI 1640 medium (Thermo Fisher Scientific, Waltham, MA, USA) supplemented with 10\% FBS (HyClone, Logan, UT, USA). HUVECs were cultured in M199 medium (Thermo Fisher Scientific) supplemented with Low Serum Growth Supplement (Thermo Fisher Scientific).

\section{Tissue microarray}

A total of 185 patients with cervical cancer were included in this analysis. All patients were diagnosed and managed at Beijing Chaoyang Hospital, affiliated to Capital Medical University, between January 2000 and September 2015. For each patient, H\&E-stained slides were used to mark the primary tumor location. One tissue cylinder (1 mm diameter) per sample was obtained and transferred into a recipient paraffin block using a manual tissue arrayer.

Five micrometer sections of TMA blocks were dewaxed in xylol for 15 minutes, rehydrated using graded alcohol and finally washed using PBS. Sections were incubated in $3 \%$ $\mathrm{H}_{2} \mathrm{O}_{2}$ (diluted in methyl alcohol) for 30 minutes to inhibit endogenous peroxidase. Antigen retrieval was performed by microwaving samples in citric acid buffer ( $\mathrm{pH}$ 6.0). Then, sections were incubated with $5 \%$ BSA (w/v) for 1 hour at room temperature. TMA sections were incubated overnight at $4{ }^{\circ} \mathrm{C}$ with rabbit anti-FPR1 (Abcam, MA, USA) antibody diluent in 5\% BSA (w/v). Incubation with secondary antibody was performed using HRP-labeled goat anti-rabbit antibody for 1 hour at room temperature, followed by treatment with $500 \mu \mathrm{L}$ DAB for 1 minute. Sections were stained with H\&E for 30 seconds and then dehydrated and mounted according to the standard operating procedures. Staining scores were calculated using Image Pro Plus 6.0.

$\mathrm{H}-\mathrm{SCORE}=\sum(\mathrm{PI} \times \mathrm{I})=($ percentage of cells of weak intensity $\times 1)+($ percentage of cells of moderate intensity

$\times 2)+($ percentage of cells of strong intensity $\times 3)$

Silencing of FPR1 expression in SiHa cells by siRNA technology was used to knock down FPR1 in vitro. siRNAs against FPR1 and corresponding scrambled siRNAs (GenePharma, Shanghai, China) were transfected into SiHa cells using Lipofectamine 2000 (Thermo Fisher Scientific) for 48 hours. The following siRNA sequences were used: siFPR1, sense: 5'-CCTGCATATCATCACTTATTT-3', antisense: 5'-ATAAGTGATGATATGCAGGTT-3'.

\section{Flow cytometry analysis}

For apoptosis analysis, cells were stained with propidium iodide (PI) and Annexin V-phycoerythrin for 15 minutes at room temperature in the dark and then analyzed on a FACSCalibur Flow Cytometer (BD Biosciences, San Jose, CA, USA). For cell cycle analysis, cells were stained with PI for 45 minutes at room temperature in the dark and then analyzed on a FACSCalibur Flow Cytometer. 


\section{Production of lentiviral particles}

Two groups of fragments, separately containing FPR1 shRNA (GCTATCTCTTCCTGGATAT) and control (scramble shRNA; TTCTCCGAACGTGTCACGT), were cloned into the GV248 plasmid (Genechem Co., Ltd, Shanghai, China). Then, GV248 was cotransfected with other packaging plasmids into HEK293T cells using Lipofectamine 2000, and viral particles were collected 48 hours after transfection.

\section{FPRI stable knockdown in $\mathrm{SiHa}$ cells}

For knockdown assays, SiHa cells were infected with a lentiviral vector carrying FPR1 shRNA or the scrambled sequence at an MOI of 30. After 72 hours of infection, cells infected with lentiviral vectors encoding enhanced green fluorescent protein (eGFP) were observed via florescence microscopy to assess transduction efficiency and eGFP expression. Cells with $80 \%$ infection efficiency and better proliferative ability were selected and expanded through puromycin treatment. The efficiency of silencing and amplification of FPR1 was confirmed by reverse transcription PCR (RT-PCR).

\section{RNA extraction and reverse transcription PCR}

Total RNA was extracted from cells or placental tissues using TRIzol (Thermo Fisher Scientific) following the manufacturer's instruction. Reverse transcription was performed with $2 \mu \mathrm{g}$ of RNA using a PrimeScript RT reagent kit with gDNA eraser (Takara Bio Inc., Dalian, China). RT-PCR was performed on a Roche Light Cycler 480 II detection system (Hoffman-La Roche Ltd., Basel, Switzerland). cDNA detection was carried out following the instructions of the SYBR $^{\circledR}$ Premix Ex Taq ${ }^{\mathrm{TM}}$ kit (Takara Bio Inc.), and reactions were carried out in duplicate at $95^{\circ} \mathrm{C}$ for 30 seconds, followed by 40 cycles of $95^{\circ} \mathrm{C}$ for 5 seconds, $60^{\circ} \mathrm{C}$ for 31 seconds. Relative FPR1 expression levels were normalized to GAPDH. The primers used for real-time PCR were as follows: FPR1, 5'-GCTCCTCACATTGCCAGTTAT-3' (forward) and 5'-CGTTGGTCCAGGGCGAAAA-3' (reverse); E-cadherin: 5'-GAGAGCTACACGTTCACGG-3' (forward) and $5^{\prime}$-GGGTGTCGAGGGAAAAATAGG-3' (reverse); $\beta$-catenin: 5'-CCCAGCGTCGTCTGCTTTA-3' (forward) and $5^{\prime}$-CGATTCGCTCTCCCCGTAAC-3' (reverse); Vimentin: 5'-GCCCTAGACGAACTGGGTC-3' (forward) and $5^{\prime}$-GGCTGCAACTGCCTAATGAG-3' (reverse); MMP9: 5'-TGTACCGCTATGGTTACACTCG-3' (forward) and 5'-GGCAGGGACAGTTGCTTCT-3' (reverse); Snail: $5^{\prime}$-TCGGAAGCCTAACTACAGCGA-3' (forward) and $5^{\prime}$-AGATGAGCATTGGCAGCGAG-3' (reverse);
Twist: 5'-GCCTAGAGTTGCCGACTTATG-3' (forward) and $5^{\prime}$-TGCGTTTCCTGTTAAGGTAGC-3' (reverse); and GAPDH, 5'-AAGGTCATCCCTGAGCTGAAC-3' (forward) and $5^{\prime}$-ACGCCTGCTTCACCACCTTCT-3' (reverse). Relative mRNA levels were calculated using the $2^{-\triangle C T}$ method.

\section{Western blotting}

Nuclear and cytoplasmic proteins were extracted following the manufacturer's instructions using the Cytoplasm protein/ Nucleoprotein extraction kit (Beyotime Institute of Biotechnology, Haimen, China). Equal amounts $(30 \mu \mathrm{g})$ of cellular extracts were separated via $10 \%$ SDS-PAGE. Proteins were transferred to nitrocellulose membranes (EMD Millipore, Billerica, MA, USA) and incubated overnight with specific antibodies, followed by incubation with specific HRP-conjugated secondary antibodies. Films were scanned using a calibrated Bio-Rad GS 800 densitometer, and signals were analyzed

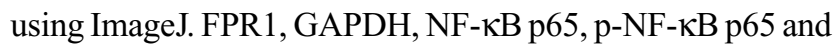
Lamin $\mathrm{B}$ antibodies were obtained from Abcam.

\section{IHC analysis}

After the experiments ended, tumor tissues were isolated and fixed in paraformaldehyde for 24 hours. Paraffin-embedded sections were stained with H\&E. The FPR1, IL-6, IL-8, CD31 and Ki67 monoclonal antibodies (Abcam) were diluted (1:100) in 5\% BSA. Cleaved caspase-3 antibody was diluted (1:50) in 5\% BSA. HRP-labeled goat anti-rat IgG antibody was used as the secondary antibody, and staining was performed with a solution containing DAB.

\section{Measurement of IL-6/8 in conditioned medium by ELISA assay}

Cells were plated at $5 \times 10^{6}$ cells $/$ well in a six-well plate, and cell culture supernatants were collected after 48 hours of culture. Then, IL- 6 and IL-8 concentrations were measured using ELISA kits according to the manufacturer's instructions (Thermo Fisher Scientific, Waltham, MA, USA).

\section{Transwell invasion assays}

Invasion assays were conducted in 24-well fitted inserts with membranes ( 8 - $\mu \mathrm{m}$ pore size; Costar, Cambridge, MA, USA) as reported previously. SiHa cells were trypsinized and seeded into transwell inserts precoated with $40 \mu \mathrm{g} / \mathrm{mL}$ Matrigel (BD Biosciences) at $5 \times 10^{4}$ cells per insert. The top chambers contained RPMI 1640 medium supplemented with $0.1 \%$ FBS, and the lower chambers were loaded with RPMI 1640 medium plus $10 \%$ FBS. After incubation for 30 hours, the cells were fixed and stained with crystal violet. Cells on 
the upper surface of the membrane were completely removed, and the number of stained cells at the lower surface of the membrane was counted on a light microscope.

\section{Wound-healing assays}

A total of $3 \times 10^{6} \mathrm{SiHa}$ cells were mixed and plated in sixwell plates. When the cells reached $80 \%$ confluence, a $200 \mu \mathrm{L}$ pipette tip was used to scratch and create a gap in the monolayer. N-formyl-methionyl-leucyl-phenylalanine (fMLF) (Sigma-Aldrich Co., St Louis, MO, USA) was added to the medium at the indicated concentrations. Cell movement phase images were subsequently captured via light microscopy every 8 hours. ImageJ software was used to analyze the mean migrated distance of the leading cells in the scratched area (gate).

\section{Xenografts}

SiHa cells were trypsinized, washed twice and resuspended in ice-cold PBS. Four-week-old female BALB/c nude mice were injected with $3 \times 10^{6}$ cells in $100 \mathrm{~mL}$ PBS. Tumor volumes were calculated according to the following formula: $\mathrm{V}=0.5 \times \mathrm{D} \times \mathrm{d} \times \mathrm{d}$, where $\mathrm{D}$ represents length and $\mathrm{d}$ represents the width of tumors measured by a digital caliper. Mice were sacrificed by $\mathrm{CO}_{2}$, and tumors were isolated and further processed. Animal care was provided in accordance with the Guide for the Care and Use of Laboratory Animals.

\section{Statistical analyses}

SPSS version 22.0 (IBM Corporation, Armonk, NY, USA) was used for the statistical analyses. All quantitative data were expressed as mean $\pm \mathrm{SD}$. Independent-samples $t$-tests were used for comparisons of variables between groups. Correlations between the detected markers were assessed using Spearman's rank correlation. $P$-values of $<0.05$ were considered statistically significant.

\section{Ethics statement}

The present study was approved by the ethics committee of Beijing Chaoyang Hospital, affiliated to Capital Medical University. All patients whose tissues were used in this study provided written informed consent in accordance with the Declaration of Helsinki. Study protocols were carried out in accordance with approved guidelines. All animal studies were carried out in accordance with the recommendations in the Guide for the Management Ordinance of Laboratory Animals of the Society of Laboratory Animals. Experimental protocols were approved by Beijing Chaoyang Hospital, affiliated to Capital Medical University. All surgical procedures were performed under anesthesia, and every effort was made to minimize suffering.

\section{Results}

\section{FPRI expression in human cervical cancer tissues}

We performed TMA analysis to establish the relationship between FPR1 expression levels in cervical cancer tissues and different pathological stages. Table 1 summarizes the clinical characteristics of the 185 cervical cancer patients involved in this study. According to the cervical cancer guide from the National Comprehensive Cancer Network, advanced-stage cervical cancer includes patients from stage IIB to IVA. We therefore divided the patients in this study into two groups: advanced stage (IIB-IVA) and early stage (I-IIA). Advancedstage cervical cancer patients had significantly higher FPR1 staining $\mathrm{H}$-scores than early stage cervical cancer patients $(P<0.001$; Table 2). Next, we compared FPR1 expression levels among cervical cancer patients with different overall

Table I Clinical characteristics of patients involved in this study

\begin{tabular}{|l|l|l|}
\hline Characteristics & $\begin{array}{l}\text { No. of patients } \\
(\mathbf{N}=\mathbf{1 8 5})\end{array}$ & $\%$ \\
\hline Age, years & & \\
$\leq 40$ & 60 & 32.4 \\
$41-50$ & 74 & 40.0 \\
$5 I-60$ & 34 & 18.4 \\
$61-70$ & 14 & 7.6 \\
$>70$ & 3 & 1.6 \\
Preoperative chemotherapy & 70 & \\
Yes & 115 & 37.8 \\
No & & 62.2 \\
Pathological stage & 129 & \\
I-IIA & 56 & 69.7 \\
IIB-IV & 155 & 30.3 \\
Lymphatic metastasis & 30 & 83.8 \\
No & & 16.2 \\
Yes & 59 & 31.9 \\
Differentiation level & 55 & 29.7 \\
High & 71 & 38.4 \\
Middle & & 10.8 \\
Low & 20 & 89.2 \\
OS & 165 & \\
OS $<5$ & & \\
OS $\geq 5$ & & \\
\hline
\end{tabular}

Abbreviation: OS, overall survival.

Table 2 FPR I staining $\mathrm{H}$-score in advanced-stage cancer patients is significantly higher than that in early stage cervical cancer patients

\begin{tabular}{|l|l|l|l|}
\hline Pathological stage & I-IIA & IIB-IV & \multirow{2}{*}{-value $^{\mathbf{a}}$} \\
\cline { 1 - 3 } No. of patients & $\mathbf{N}=\mathbf{I 2 9}$ & $\mathbf{N}=\mathbf{5 6}$ & \\
\hline FRPI & $130.8 \pm 34.2$ & $209.6 \pm 31.7$ & $<0.00$ I \\
\hline
\end{tabular}

Note: ${ }^{\mathrm{T} T w o-s a m p l e ~ r a n k-s u m ~ t e s t ~ u s i n g ~ S P S S ~ 22.0 . ~}$ 
survival times. The data demonstrated that high FPR1 expression levels are related to poor prognosis $(P<0.001$; Table 3$)$.

\section{FPRI protein and mRNA expression in human cervical cancer cell lines}

To explore the biological function of FPR1 in cervical cancer development, two established human cervical cancer cell lines, SiHa and HeLa, were used in our experiments. Western blotting (Figure 1A and B) and RT-PCR (Figure 1C) showed that both SiHa and HeLa cells expressed FPR1, with SiHa cells expressing higher levels of FPR1 than HeLa cells $(P<0.01)$.

\section{FPR I promotes human cervical cancer cell invasion, migration and proliferation}

We chose SiHa cells to test the biological function of FPR1 in human cervical cancer cells. First, we designed siRNA oligos to silence FPR1 expression in SiHa cells. As shown in Figure $2 \mathrm{~A}$ and $\mathrm{B}$, compared with control siRNA, the specific siRNA suppressed FPR1 expression in SiHa cells $(P<0.01)$. Next, we transfected si-FPR1 oligos into $\mathrm{SiHa}$ cells to explore the role of FPR1. Silencing of FPR1 expression using specific

Table 3 High FPRI staining $\mathrm{H}$-score indicates poor prognosis

\begin{tabular}{|l|l|l|l|}
\hline OS & $\begin{array}{l}\text { OS } \\
<5 \text { years }\end{array}$ & $\begin{array}{l}\text { OS } \\
\geq 5 \text { years }\end{array}$ & \multirow{2}{*}{-value $^{\mathbf{a}}$} \\
\cline { 1 - 3 } No. of patients & $\mathbf{N}=\mathbf{2 0}$ & $\mathbf{N}=165$ & \\
\hline FRPI & $142.2 \pm 39.6$ & $169.0 \pm 41.7$ & $<0.001$ \\
\hline
\end{tabular}

Note: ${ }^{\mathrm{T}}$ Two-sample rank-sum test using SPSS 22.0.

Abbreviation: OS, overall survival.
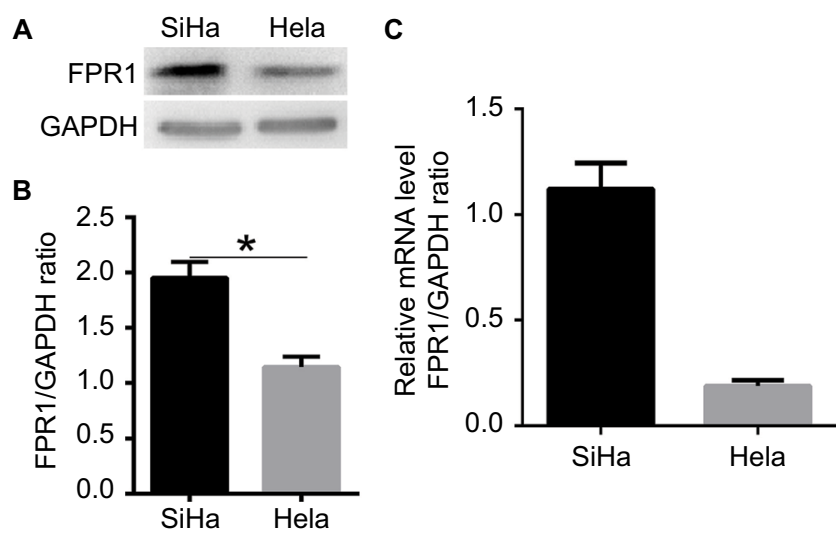

Figure I The expression of FPRI protein and mRNA in human cervical cancer cell lines.

Notes: (A, B) Western blot showing the relative FPRI expression levels in HeLa and SiHa cells. (C) RT-PCR showing the relative FPRI expression levels in HeLa and $\mathrm{SiH}$ a cells. Bar chart according to the statistical analysis based on the results of three independent experiments. Data are expressed as mean \pm SEM. $* P<0.05$

Abbreviations: RT-PCR, reverse transcription PCR; SEM, standard error of the mean.
siRNAs resulted in the inhibition of cell invasion $(P<0.01)$, especially under fMLF treatment, which is a typical FPR 1 agonist $(P<0.01$; Figure $2 \mathrm{C}$ and $\mathrm{D})$.

To more precisely examine the contribution of FPR 1 to cervical cancer cell motility and invasiveness, we used a wound-healing model by creating a gap in a confluent $\mathrm{SiHa}$ cell monolayer. Silencing of FPR1 expression inhibited cell migration $(P<0.01$; Figure $2 \mathrm{E}$ and $\mathrm{F})$. Thus, FPR1 activation promoted cell migration. SiHa cells treated with $\mathrm{fMLF}$ showed more rapid migration than vehicle-treated cells toward the center of the gap in the cell monolayer. These data demonstrated that FPR1 regulated cervical cancer cell motility.

Next, to further verify the role of FPR1 in human cervical cancers, we detected epithelial-mesenchymal transition (EMT)-related proteins and transcription factors in FPR1silenced SiHa cells. FPR1 silencing inhibited Vimentin $(P<0.05)$ and MMP9 $(P<0.05)$ expression (Figure $2 \mathrm{G})$ but promoted E-cadherin $(P<0.05)$ and $\beta$-catenin expression $(P<0.05$; Figure $2 \mathrm{G})$. FPR1 also inhibited the expression of the transcription factors such as Twist $(P<0.05)$ and Snail $(P<0.05$; Figure $2 \mathrm{H})$. Thus, silencing of FPR1 inhibited SiHa cell EMT program. Overall, we demonstrated that FPR1 promotes cervical cancer cell malignant phenotypes, which matches the poor prognosis in cervical cancer patients and cell functional assays.

We then examined whether knocking down FPR1 expression affected SiHa cell proliferation using FACS analysis. Compared with control cells, the proportion of apoptotic cells increased in FPR1 knockdown in SiHa cells. The results showed that knocking down FPR1 expression induced $\mathrm{SiHa}$ cell apoptosis (Figure 2I and J). We then examined the cell cycle by FACS analysis. We found that knocking down FPR1 expression significantly blocked the cell cycle transition from the $\mathrm{G} 1$ to $\mathrm{S}$ phase in SiHa cells (Figure 2K and L), indicating the induction of G1 arrest. Thus, FPR1 activity promotes cell proliferation.

\section{FPR I mediates NF- $\kappa B$ activation in cervical cancer cells}

Since FPR1 is a typical GPCR involved in many signaling pathways, especially inflammatory signaling, we stimulated serum-starved SiHa cells with $100 \mathrm{nM}$ fMLF and tested NF- $\kappa B$ activation in cells with or without FPR1 knockdown. FPR1 knockdown inhibited NF- $\kappa B$ phosphorylation, suggesting that $\mathrm{AMLF}$ promotes NF- $\mathrm{KB}$ phosphorylation through FPR1 (Figure 3A). Further analysis showed that FPR1 silencing decreased NF- $\mathrm{\kappa B}$ subunit P65 expression in the nucleus 


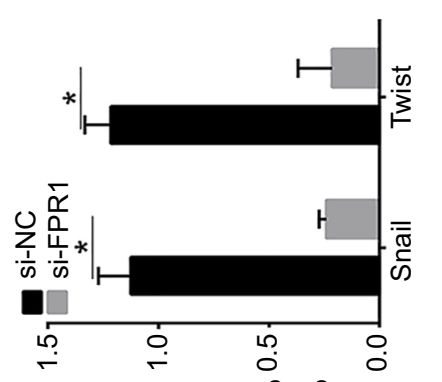

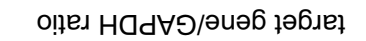

I әлә| $\forall$ Ny山 әл!ฺए|әу

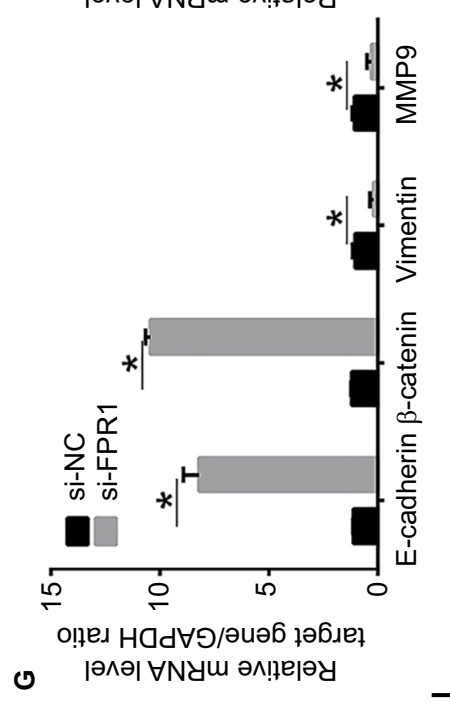

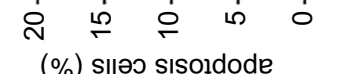

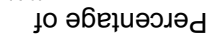
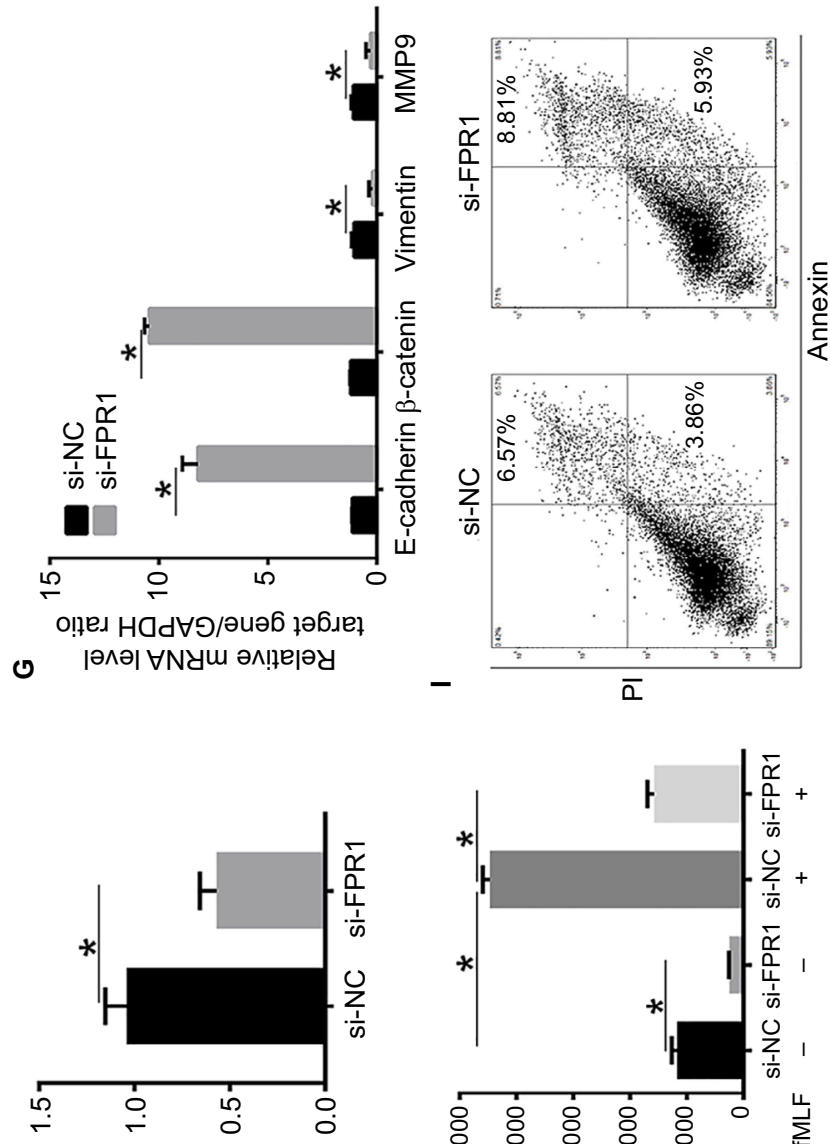

$\boldsymbol{m}$

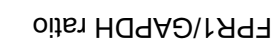
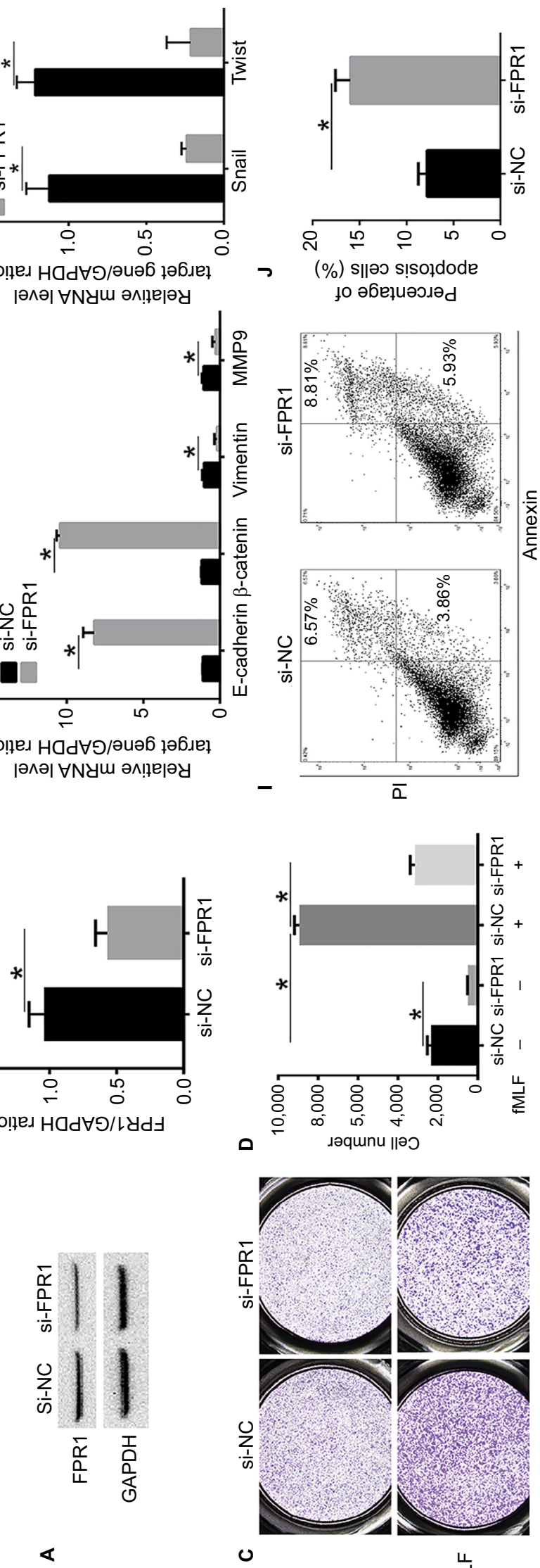

\lrcorner

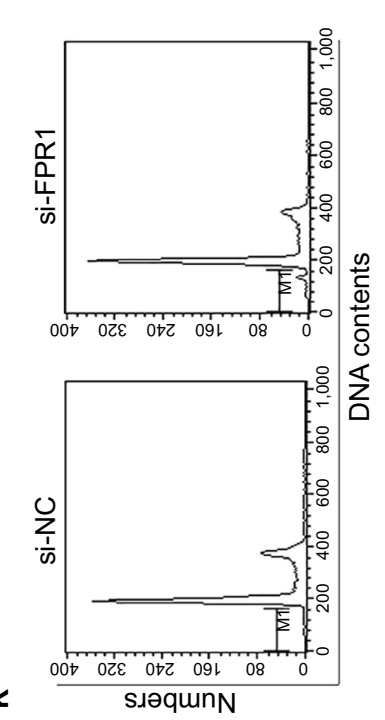

$\underline{1}$
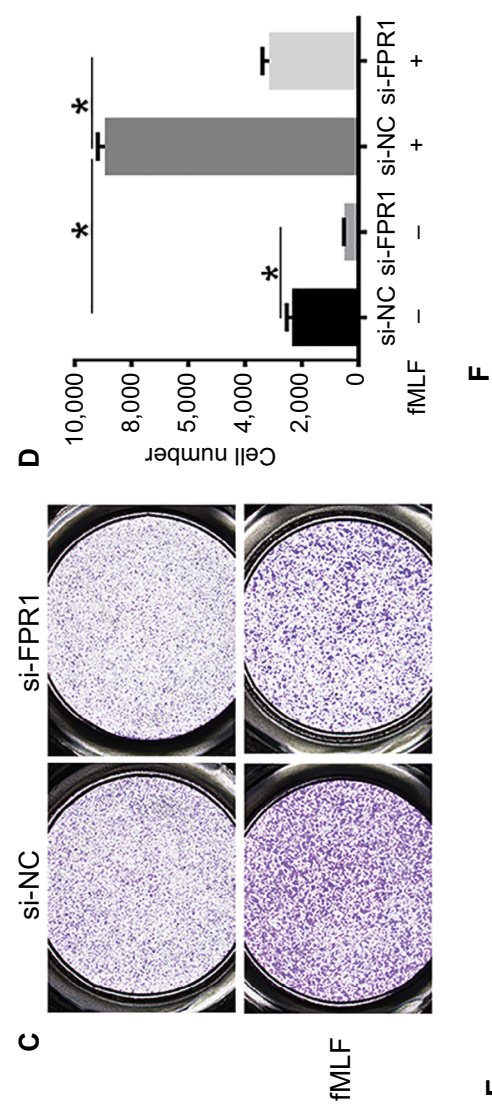

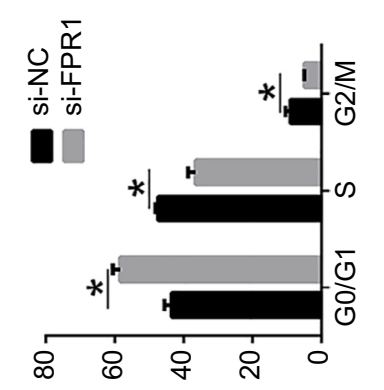

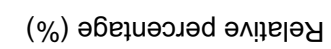

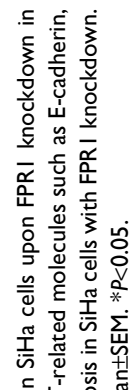

它它芯

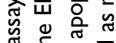

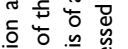

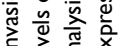

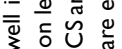

氙范

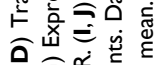

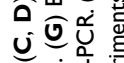

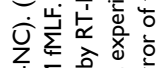

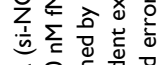

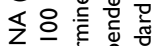

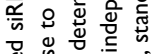

造施造出

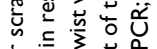

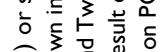

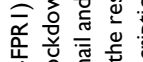

景立战

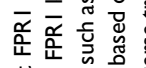

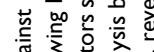

品

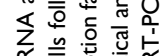

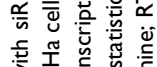

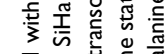

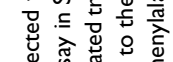

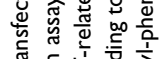

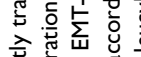

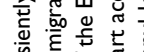

牙 $\overline{\overline{0}}$ 呵

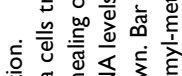

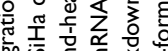

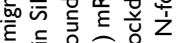

둘

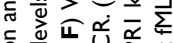

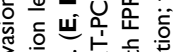

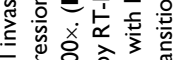

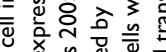

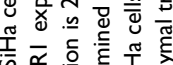

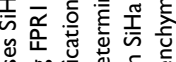

⿹े

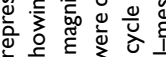

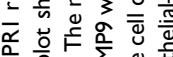

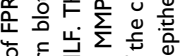

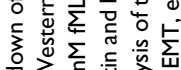

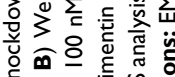

文过方出

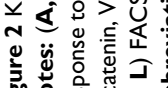


A
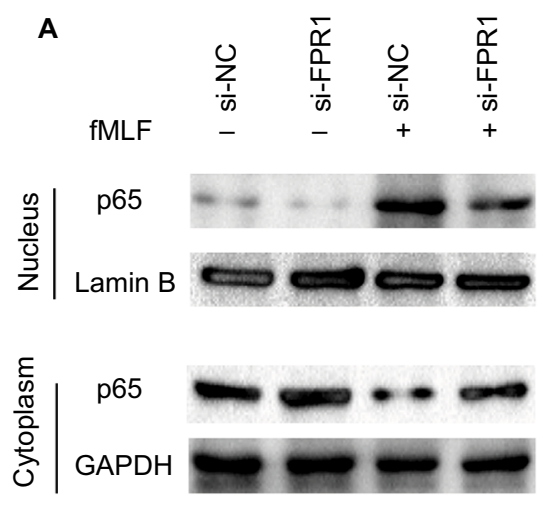

\begin{tabular}{c|r}
$\overline{\bar{d}}$ & FPR1 \\
ঠ & p65 \\
\hline O & p-p65 \\
\} $&{\text { GAPDH }}$
\end{tabular} $\frac{\bar{\alpha}}{0.0}$

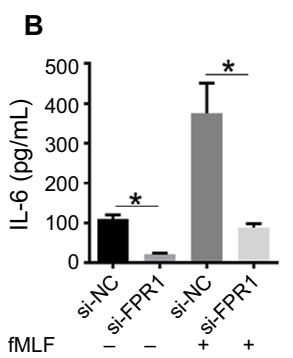

E

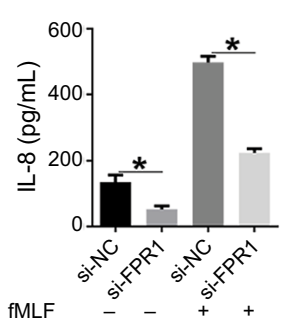

C SiHa supernatant (48h)

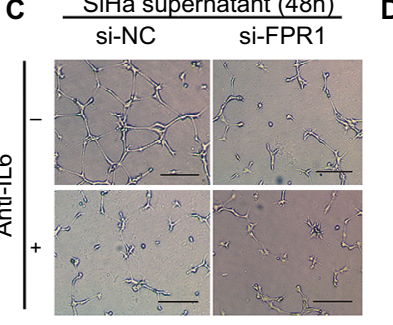

F SiHa supernatant (48h)

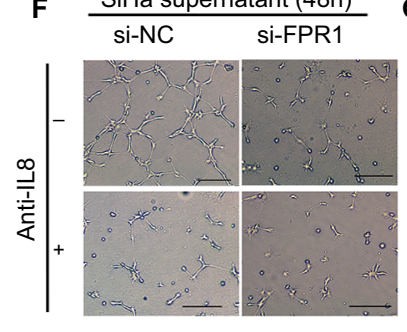

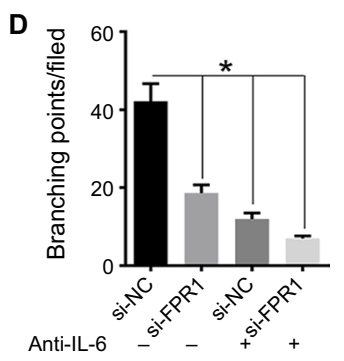

G

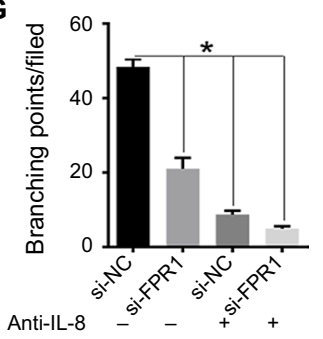

Figure 3 FPRI mediated NF-KB activation and IL-6/8 production by SiHa cells.

Notes: (A) Effects of FPRI knockdown on the nuclear translocation and activation of P65. NF- $\mathrm{KB}$ expression in SiHa cells stimulated with $100 \mathrm{nM}$ fMLF for 20 minutes was examined by Western blotting. (B, E) IL-6 and IL-8 levels in the supernatants of SiHa cells treated with $100 \mathrm{nM}$ fMLF, as measured by ELISA. (C, D) HUVEC tube formation assays on Matrigel. Culture supernatants from SiHa cells treated with $100 \mathrm{nM}$ fMLF for 48 hours were incubated with anti-IL-6 antibody (C) or IgG (-) (each at I mg/mL) for 2 hours. HUVECs were then mixed with the supernatants and examined for tubule formation on Matrigel after 4 hours. (F, G) Culture supernatants from SiHa cells treated with $100 \mathrm{nM}$ fMLF for 48 hours were incubated with an anti-IL-8 antibody $(\mathbf{C})$ or $\operatorname{lgG}(-)$ (each at I mg/mL) for 2 hours. HUVECs were then mixed with the supernatants and examined for tubule formation on Matrigel after 4 hours. Bar chart according to the statistical analysis based on the result of three independent experiments. Data are expressed as mean $\pm S E M$. $* P<0.05$.

Abbreviations: fMLF, N-formyl-methionyl-leucyl-phenylalanine; HUVECs, human umbilical vein endothelial cells; SEM, standard error of the mean.

and increased P65 expression in the cytoplasm (Figure 3A). In addition, the phosphorylated (ie, active) P65 levels were reduced in FPR1-silenced SiHa cells (Figure 3A). These results suggested that the nuclear translocation and activation of P65 were inhibited in FPR1-silenced SiHa cells.

\section{FPRI activation enhances angiogenesis by promoting IL-6 and IL-8 production}

Then, we examined the inflammatory factors such as IL-6 and IL-8 in human cervical cancer cells. FPR1 knockdown inhibited IL-6 and IL-8 secretion, particularly upon treatment with fMLF (Figure 3B and E). Furthermore, because IL-6 and IL-8 can promote angiogenesis in many tumors, we next used HUVEC tube formation assays to evaluate FPR1 function in angiogenesis. We collected cell culture supernatants from SiHa cells with or without FPR1 knockdown, followed by incubation with anti-IL-6 or anti-IL-8 antibody. The experiments showed that angiogenesis was inhibited by the addition of a monoclonal antibody against human IL-6/8. Thus, IL-6 and IL- 8 in conditioned supernatants from FPR1-activated SiHa cells induce endothelial cells to form capillary-like structures, which is important in tumorigenesis (Figure 3C, D, F and G).

\section{FPRI promotes cervical cancer development}

We used a lentivirus-shRNA system to induce FPR1 knockdown in SiHa cells, with lentivirus-shScramble RNAinfected SiHa cells serving as a control group. FPR1 mRNA expression level was stably repressed in the knockdown cells (Figure 4A and B). To investigate the function of FPR1 in cell proliferation, Cell Counting Kit- 8 assays were then carried out. FPR1 deficiency inhibited SiHa cell proliferation (Figure 4C).

To assess the role of FPR1 in cervical cancer development in vivo, two groups of nude mice, with 10 animals in each group, were injected with lentivirus FPR1 knockdown and control cells into the subcutaneous tissue in the upper back, and tumor size was monitored every 5 days. Tests were terminated, and tumor sampling was performed when the tumor size in control animals reached a volume of $0.5-1$ $\mathrm{cm}^{3}$. Compared with the control group, FPR1 knockdown in SiHa cells exhibited suppressed tumor formation ability (Figure 4D and E). The tumor growth rate of the FPR1 deficiency group was significantly lower than that of the normal control group (Figure 4E). Thus, FPR1 promotes cervical cancer development. 
A

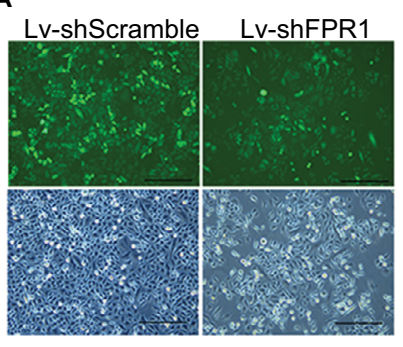

D

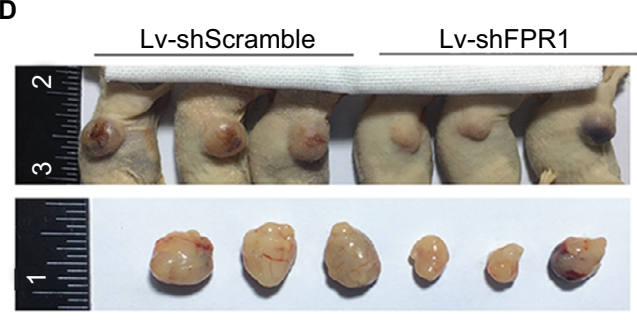

B

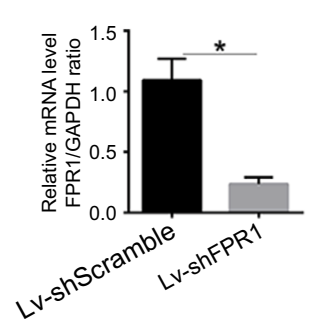

C

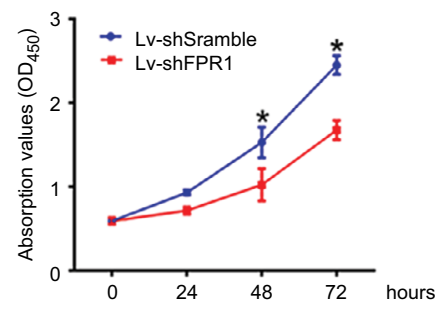

E

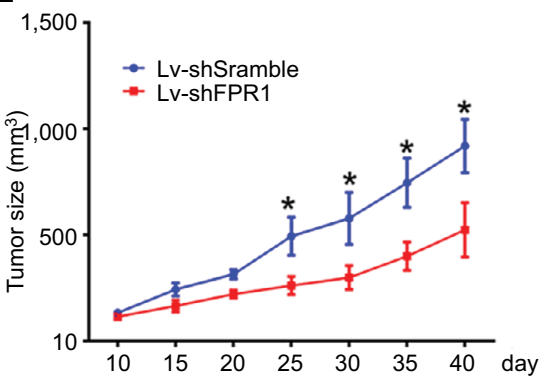

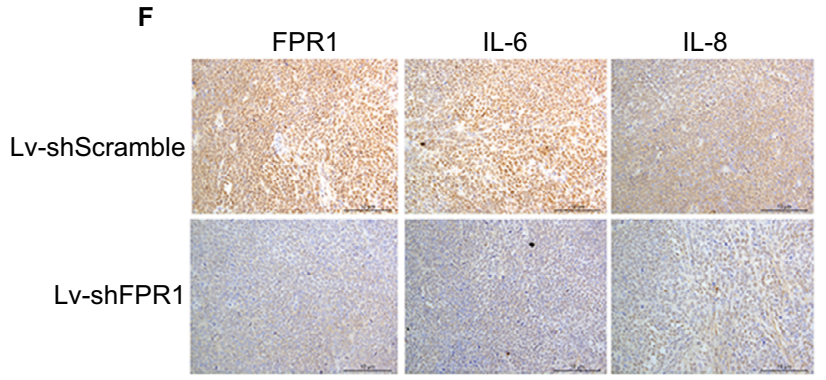

G

HE

CD31

Ki-67

Cleaved
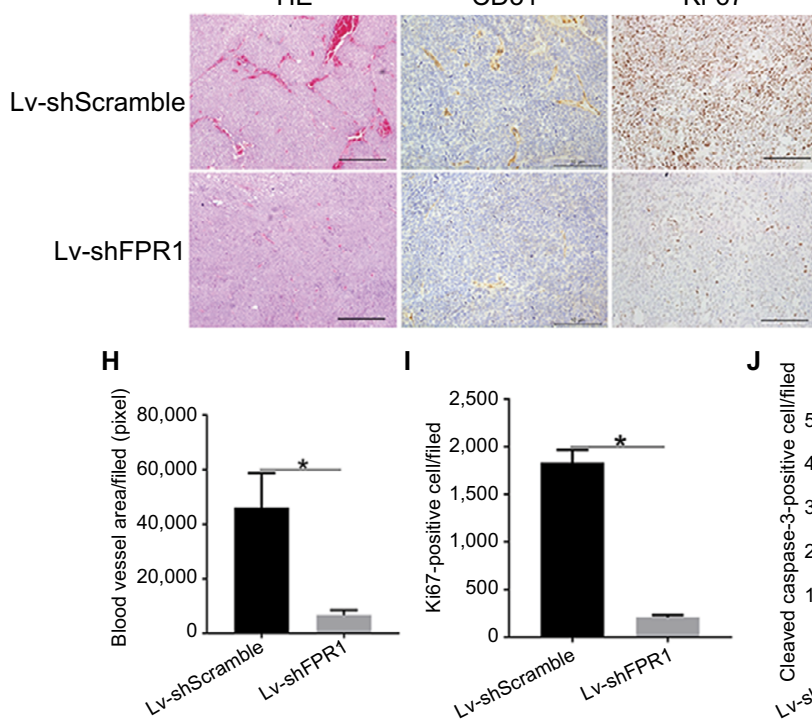

caspase 3
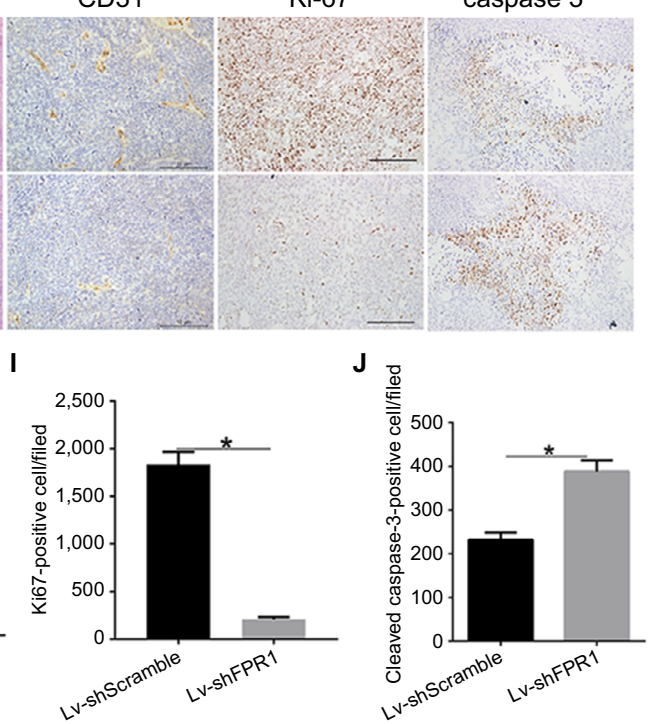

Figure 4 The effect of FPRI knockdown on SiHa cell tumorigenicity in nude mice.

Notes: (A) Construction of $\mathrm{SiH}$ a cells with stable FPRI knockdown using a lentivirus-shRNA system. The magnification is 200x. (B) RT-PCR showing the relative expression levels of FPRI mRNA in SiHa cells stably transfected with FPRI lentivirus-shFPRI; lentivirus-shScramble was used as a control. (C) Growth curves of shRNA-transfected $\mathrm{SiHa}$ cells. Cell growth was measured using CCK-8 assays. (D, E) Tumor formation assays. SiHa cells were injected subcutaneously into the upper back of nude mice (I0 mice per group). Mice were examined for tumor formation every 5 days. (F) IHC staining showing FPRI, IL-6 and IL-8 expression in tumor tissue. The magnification is 200×. (G) H\&E and IHC staining of tumor structure. The magnification is $200 \times$. The vascular epithelial cell marker CD3I, the ubiquitous cell cycle marker Ki67 and the apoptosis marker cleaved caspase-3 were stained to evaluate neovascularization, proliferation and apoptosis, respectively, in tumor tissue. (H-J) Bar chart showing the area of neovascularization in tumor tissue and the numbers of Ki67-positive and cleaved caspase-3-positive cells. Data are expressed as mean $\pm S E M$. $* P<0.05$.

Abbreviations: CCK-8, Cell Counting Kit-8; IHC, immunohistochemistry; RT-PCR, reverse transcription PCR; SEM, standard error of the mean. 
Following these experiments, tumor tissues were isolated and fixed for histological analysis to detect for FPR1, IL-6 and IL-8 expression levels (Figure 4F). As expected, IL-6 and IL-8 expressions were repressed in FPR1-deficient cells. We clearly observed fewer blood cells in tumors from the FPR1 knockdown group than in the control group by H\&E staining (Figure 4G). To further verify the role of FPR1 in angiogenesis, we used the epithelial cell marker CD31 to indicate blood vessels in the tumor. The blood vessel area was significantly lower in the FPR1 knockdown cells than in the control group $(P<0.01)$. Thus, FPR1 deficiency inhibited tumor angiogenesis (Figure $4 \mathrm{G}$ and $\mathrm{H}$ ). This finding is consistent with the results of our in vitro experiments.

To understand whether FPR1 has any effect on tumor formation, we analyzed the proliferation and apoptosis ability of tumors with FRP1 knockdown. For this purpose, we stained cells for the ubiquitous cell cycle marker Ki67 and apoptosis marker cleaved caspase-3. IHC analysis revealed lower numbers of $\mathrm{Ki} 67^{+}$nuclei in FPR1 knockdown tumors than in the control group (Figure $4 \mathrm{G}$ and I), whereas the number of cleaved caspase $-3^{+}$cells increased significantly in the FPR1 knockdown group, indicating that FPR1 inhibits apoptosis in cervical cancer cells (Figure 4G and J).

\section{Discussion}

FPR1 is reported to be overexpressed in a wide variety of tumors and to be involved in cancer progression. ${ }^{7,8,25,26}$ Database analyses revealed that FPR1 expression levels are upregulated in malignant glioma cells, colon cancer, lung cancer and hepatocellular carcinoma cells compared with those in normal controls. ${ }^{27-30}$ According to our results, FPR1 was also overexpressed in tumor tissues from terminal-stage cervical cancer patients and from patients with poor prognosis. These observations indicated that FPR 1 expression levels may serve as a diagnostic marker for cervical cancer. Furthermore, FPR1 silencing inhibited cervical cancer cell tumor features such as invasion and migration, suggesting that high FPR1 expression may facilitate cervical cancer development and also explaining the link between high FPR 1 levels and poor prognoses in cervical cancer patients. These findings demonstrate a potentially important role for FPR1 in the diagnosis and prognosis of cervical cancer patients. We therefore hypothesized that FPR1 may function as an oncogene involved in the tumorigenesis and progression of cervical cancer. To test this hypothesis, we performed a series of in vitro and in vivo assays. Our results showed that knocking down FPR1 inhibited malignant phenotypes such as proliferation, EMT, migration and invasion. These data suggested that FPR1 is indeed an oncogene that plays a vital role in cervical cancer progression.

FPR1 localizes to the surface and can be activated by Anx-A1 secreted by cancer cells and normal cells. Activated FPR1 induces the activation of a series of regulatory molecules, including p38, MAPK and ERK1/2, as well as many transcription factors, such as NF- $\mathrm{KB}$, STAT3 and HIF-1 $\alpha$, to promote cancer cell proliferation, invasion and migration. ${ }^{11,13,31,32}$ All the data demonstrate that FPR1 agonists within tumor cell microenvironment induce several vital signaling pathways that promote tumorigenesis. We focused on the activation and NF- $\kappa \mathrm{B}$ translocation into the cell nucleus to regulate the synthesis and secretion of inflammatory factors. Our results showed that FPR1-activated NF-אB signaling pathways involved in cervical cancer development.

We then examined the expression levels of the typical inflammatory factors such as IL-6 and IL-8. IL-6 and IL-8 are known to be directly implicated in the onset of a wide variety of tumors and are considered to be tumor growth factors. ${ }^{33,34}$ IL-6 and IL-8 expression levels are significantly elevated in tissues from numerous cancers compared with those in healthy adjacent tissues. ${ }^{35-37}$ IL-6 and IL-8 are also involved in cervical cancer progression, which is consistent with our results. The role of inflammatory responses in tumorigenesis is very complicated. IL-6 also plays an important role in the proliferation and apoptosis of tumor cells. IL- 6 binds to GP130 to activate various signal transduction pathways. IL-6/STAT3 activation is involved in cell apoptosis (Bcl-2), angiogenesis (VEGF), cell invasion and migration (MMP2/9) and particularly cell inflammation (IL-17). .4,38,39 $^{2}$

Our experiments showed that IL-6 and IL-8 expression levels in cervical cancer cells are regulated by FPR1-related signaling. Thus, cervical cancer tissues produce large quantities of IL- 6 and IL- 8 that can induce reactions throughout the body, especially in the local cancer microenvironment. Both IL-6 and IL-8 perform multiple functions in the onset and development of cancer. IL- 6 upregulation in the cervical cancer microenvironment can induce cancer cell proliferation and invasion and promote angiogenesis in tumor tissue. IL-8 is upregulated in cervical cancer tissues and is associated with cervical cancer cell proliferation and migration. These findings demonstrate that IL-6 and IL-8 play a vital role in cervical cancer progression. Nevertheless, the specific role of IL- 6 and IL-8 in the pathogenesis of cervical cancer remains elusive. IL-6 and IL-8 generally thought to induce VEGF expression in angiogenesis, and our results indicated that IL- 6 and IL-8 may facilitate the development of cervical cancer by modulating tumor angiogenesis. 


\section{Conclusion}

High FPR1 expression levels were associated with advanced stage and poor prognosis in cervical cancer patients. FPR1 activation induced NF- $\mathrm{KB}$ nuclear translocation and promoted the development of cervical cancer by upregulating IL-6 and IL-8 expression levels. Inhibition of FPR 1 activity may therefore have potential therapeutic value in patients with cervical cancer.

\section{Acknowledgment}

This study was supported in part by the Beijing Municipal Administration of Hospitals Clinical Medicine Development of Special Funding Support and 2012DFR30490 (ZZ).

\section{Disclosure}

The authors report no conflicts of interest in this work.

\section{References}

1. Islami F, Torre LA, Drope JM, Ward EM, Jemal A. Global cancer in women: cancer control priorities. Cancer Epidemiol Biomarkers Prev. 2017;26(4):458-470.

2. Torre LA, Bray F, Siegel RL, Ferlay J, Lortet-Tieulent J, Jemal A. Global cancer statistics, 2012. CA Cancer J Clin. 2015;65(2):87-108.

3. Salicrú SR, de La Torre JF, Gil-Moreno A. The surgical management of early-stage cervical cancer. Curr Opin Obstet Gynecol. 2013;25(4):312-319.

4. Marshall C, Rajdev MA, Somarouthu B, Ramaiya NH, Alessandrino F. Overview of systemic treatment in recurrent and advanced cervical cancer: a primer for radiologists. Abdom Radiol. 2018.

5. Liu M, Chen K, Yoshimura T, et al. Formylpeptide receptors mediate rapid neutrophil mobilization to accelerate wound healing. PLoS One. 2014;9(6):e90613.

6. Vacchelli E, Ma Y, Baracco EE, et al. Chemotherapy-induced antitumor immunity requires formyl peptide receptor 1 . Science. 2015;350(6263):972-978.

7. Belvedere R, Bizzarro V, Popolo A, et al. Role of intracellular and extracellular annexin A1 in migration and invasion of human pancreatic carcinoma cells. BMC Cancer. 2014;14:961.

8. Boer JC, van Marion DM, Joseph JV, et al. Microenvironment involved in FPR1 expression by human glioblastomas. J Neurooncol. 2015;123(1):53-63.

9. Le Y, Ye RD, Gong W, Li J, Iribarren P, Wang JM. Identification of functional domains in the formyl peptide receptor-like 1 for agonistinduced cell chemotaxis. Febs J. 2005;272(3):769-778.

10. Huang S, Chen LY, Zuraw BL, Ye RD, Pan ZK. Chemoattractant-stimulated NF-kappaB activation is dependent on the low molecular weight GTPase RhoA. J Biol Chem. 2001;276(44): 40977-40981.

11. Jo EJ, Lee HY, Kim JI, et al. Activation of formyl peptide receptor-like 1 by WKYMVm induces serine phosphorylation of STAT3, which inhibits its tyrosine phosphorylation and nuclear translocation induced by hydrogen peroxide. Life Sci. 2004;75(18):2217-2232.

12. Liu FC, Yu HP, Syu YT, et al. Honokiol suppresses formyl peptideinduced human neutrophil activation by blocking formyl peptide receptor 1. Sci Rep. 2017;7(1):6718.

13. Shi Y, Lai X, Ye L, et al. Activated niacin receptor HCA2 inhibits chemoattractant-mediated macrophage migration via $\mathrm{G} \beta \gamma / \mathrm{PKC} /$ ERK1/2 pathway and heterologous receptor desensitization. Sci Rep. $2017 ; 7: 42279$
14. Wang X, Qin W, Song M, Zhang Y, Sun B. Exogenous carbon monoxide inhibits neutrophil infiltration in LPS-induced sepsis by interfering with FPR1 via p38 MAPK but not GRK2. Oncotarget. 2016;7(23):34250-34265.

15. Singh R, Mishra MK, Aggarwal H. Inflammation, immunity, and cancer. Mediators Inflamm. 2017;2017:6027305.

16. Wu Y, Dong Y, Duan S, Zhu D, Deng L. Metabolic syndrome, inflammation, and cancer. Mediators Inflamm. 2017;2017:8259356-2.

17. Kinoshita T, Ito H, Miki C. Serum interleukin-6 level reflects the tumor proliferative activity in patients with colorectal carcinoma. Cancer. 1999;85(12):2526-2531.

18. Lima SF, Tavares MM, Macedo JL, et al. Influence of IL-6, IL-8, and TGF- $\beta 1$ gene polymorphisms on the risk of human papillomavirusinfection in women from Pernambuco, Brazil. Mem Inst Oswaldo Cruz. 2016;111(11):663-669.

19. Wei LH, Kuo ML, Chen CA, et al. Interleukin-6 in cervical cancer: the relationship with vascular endothelial growth factor. Gynecol Oncol. 2001;82(1):49-56.

20. Jia L, Li F, Shao M, et al. IL-8 is upregulated in cervical cancer tissues and is associated with the proliferation and migration of HeLa cervical cancer cells. Oncol Lett. 2018;15(1):1350-1356.

21. Tartour E, Gey A, Sastre-Garau X, et al. Analysis of interleukin 6 gene expression in cervical neoplasia using a quantitative polymerase chain reaction assay: evidence for enhanced interleukin 6 gene expression in invasive carcinoma. Cancer Res. 1994;54(23):6243-6248.

22. Anglesio MS, George J, Kulbe H, et al. IL6-STAT3-HIF signaling and therapeutic response to the angiogenesis inhibitor sunitinib in ovarian clear cell cancer. Clin Cancer Res. 2011;17:2538-2548.

23. Radziejewska I, Borzym-Kluczyk M, Leszczyńska K, Wosek J, Bielawska A. Lotus tetragonolobus and Maackia amurensis lectins influence phosphoIאB $\alpha$, IL-8, Lewis b and $\mathrm{H}$ type 1 glycoforms levels in $\mathrm{H}$. pylori infected CRL-1739 gastric cancer cells. Adv Med Sci. 2018;63(1):205-211.

24. Zou M, Zhang X, Xu C. IL6-induced metastasis modulators p-STAT3, MMP-2 and MMP-9 are targets of 3,3'-diindolylmethane in ovarian cancer cells. Cell Oncol. 2016;39(1):47-57.

25. Cheng TY, Wu MS, Lin JT, et al. Formyl Peptide receptor 1 expression is associated with tumor progression and survival in gastric cancer. Anticancer Res. 2014;34(5):2223-2229.

26. Lammers KM, Chieppa M, Liu L, et al. Gliadin induces neutrophil migration via engagement of the formyl peptide receptor, FPR1. PLoS One. 2015;10(9): $\mathrm{e} 0138338$.

27. Farooq SM, Stadnyk AW. Neutrophil infiltration of the colon is independent of the FPR1 yet FPR1 deficient mice show differential susceptibilities to acute versus chronic induced colitis. Dig Dis Sci. 2012;57(7):1802-1812.

28. Morris S, Vachani A, Pass HI, et al. Whole blood FPR1 mRNA expression predicts both non-small cell and small cell lung cancer. Int $J$ Cancer. 2018;142(11):2355-2362.

29. Vecchi L, Alves Pereira Zóia M, Goss Santos T, et al. Inhibition of the AnxA1/FPR1 autocrine axis reduces MDA-MB-231 breast cancer cell growth and aggressiveness in vitro and in vivo. Biochim Biophys Acta Mol Cell Res. 2018;1865(9):1368-1382.

30. Zhang L, Wang H, Yang T, et al. Formylpeptide receptor 1 mediates the tumorigenicity of human hepatocellular carcinoma cells. Oncoimmunology. 2016;5(2):e1078055.

31. Prevete N, Liotti F, Visciano C, Marone G, Melillo RM, de Paulis A. The formyl peptide receptor 1 exerts a tumor suppressor function in human gastric cancer by inhibiting angiogenesis. Oncogene. 2015;34(29):3826-3838.

32. Zhang X, Wang T, Yuan ZC, et al. Mitochondrial peptides cause proinflammatory responses in the alveolar epithelium via FPR-1, MAPKs, and AKT: a potential mechanism involved in acute lung injury. Am $J$ Physiol Lung Cell Mol Physiol. 2018.

33. Ahmad N, Ammar A, Storr SJ, et al. IL-6 and IL-10 are associated with good prognosis in early stage invasive breast cancer patients. Cancer Immunol Immunother. 2018;67(4):537-549. 
34. Ding S, Tang Z, Jiang Y, et al. IL-8 is involved in estrogen-related receptor $\alpha$-regulated proliferation and migration of colorectal cancer cells. Dig Dis Sci. 2017;62(12):3438-3446.

35. Fu Q, Liu P, Sun X, et al. Ribonucleic acid interference knockdown of IL-6 enhances the efficacy of cisplatin in laryngeal cancer stem cells by downregulating the IL-6/STAT3/HIF1 pathway. Cancer Cell Int. 2017;17:79.

36. Kim W, Pyo J, Noh BJ, Jeong JW, Lee J, Kim JE. CCAR2 negatively regulates IL-8 production in cervical cancer cells. Oncotarget. 2018;9(1):1143-1155.

37. Sanguinete MMM, Oliveira PH, Martins-Filho A, et al. Serum IL-6 and IL-8 correlate with prognostic factors in ovarian cancer. Immunol Invest. 2017;46(7):677-688.
38. Chevrel G, Garnero P, Miossec P. Addition of interleukin 1 (IL1) and IL17 soluble receptors to a tumour necrosis factor alpha soluble receptor more effectively reduces the production of IL6 and macrophage inhibitory protein-3alpha and increases that of collagen in an in vitro model of rheumatoid synoviocyte activation. Ann Rheum Dis. 2002;61(8):730-733.

39. Grünwald B, Vandooren J, Gerg M, et al. Systemic ablation of MMP-9 triggers invasive growth and metastasis of pancreatic cancer via deregulation of IL6 expression in the bone marrow. Mol Cancer Res. 2016;14(11):1147-1158.
Cancer Management and Research

\section{Publish your work in this journal}

Cancer Management and Research is an international, peer-reviewed open access journal focusing on cancer research and the optimal use of preventative and integrated treatment interventions to achieve improved outcomes, enhanced survival and quality of life for the cancer patient The manuscript management system is completely online and includes

\section{Dovepress}

a very quick and fair peer-review system, which is all easy to use. Visit http://www.dovepress.com/testimonials.php to read real quotes from published authors. 